7-1-2005

\title{
Color Mixing in the Metering Zone of A Single Screw Extruder: Numerical Simulations and Experimental Validation
}

Kirill Alemaskin

Case Western Reserve University

Ica Manas Zloczower

Case Western Reserve University, ica.manas-zloczower@case.edu

Miron Kaufman

Cleveland State University, m.kaufman@csuohio.edu

Follow this and additional works at: https://engagedscholarship.csuohio.edu/sciphysics_facpub

Part of the Physics Commons

How does access to this work benefit you? Let us know!

\section{Publisher's Statement}

This is the accepted version of the following article: Kirill Alemaskin, Ica Manas-Zloczower and Miron Kaufman, "Color mixing in the metering zone of a single screw extruder: Numerical simulations and experimental validation," Polymer Engineering \& Science 45 (7), 1011-1020 (2005). , which has been published in final form at http://onlinelibrary.wiley.com/wol1/doi/ 10.1002/pen.20368/abstract

\section{Repository Citation}

Alemaskin, Kirill; Zloczower, Ica Manas; and Kaufman, Miron, "Color Mixing in the Metering Zone of A Single Screw Extruder: Numerical Simulations and Experimental Validation" (2005). Physics Faculty Publications. 233.

https://engagedscholarship.csuohio.edu/sciphysics_facpub/233

This Article is brought to you for free and open access by the Physics Department at EngagedScholarship@CSU. It has been accepted for inclusion in Physics Faculty Publications by an authorized administrator of EngagedScholarship@CSU. For more information, please contact library.es@csuohio.edu. 


\title{
Color Mixing in the Metering Zone of a Single Screw Extruder: Numerical Simulations and Experimental Validation
}

\author{
Kirill Alemaskin, Ica Manas-Zloczower \\ Miron Kaufman
}

\section{INTRODUCTION}

Single-screw extruders (SSEs) are widely used in industrial polymer processing. Although nowadays in polymer processing they are mostly used as pumping devices for processes such as injection or blow molding, their mixing efficiency should not be underestimated. The fundamentals of mixing in the metering zone of a conventional SSE are well described using the concept of an unwound channel with a moving upper wall, which generates a three-dimensional flow [1].

Since Maddock [2] proposed a technique of visual screw mixing analysis in the late 1950 s, known as "screw freezing" or "screw crash", many visual analyses of mixing in a SSE have been done, including those devoted to mixing in a melt conveying zone [3-8]. As Benkreira et al. [9] showed, mixing in a conventional Archimedean screw primarily occurs during the melting phase and improves little thereafter.

Periodic reorientation of flow inside the channel may enhance mixing by the generation of chaotic patterns. Therefore inclusion of periodic obstacles in the channel, such as pins $[10,11]$ or spatial periodic barriers in chaotic mixers [12], can enhance the mixing efficiency of a conventional screw. Other more complex designs allow imparting substantial shear strain as well as providing material reorientation [13-19].

Numerical simulations provide an opportunity to study the mechanics of flow and mixing in such complex geometries without performing the actual experiments. Somers et al. [20] has studied temperature distributions in the energy transfer screw employing a finite element simulation. Twodimensional (2D) and three-dimensional (3D) numerical studies of mixing efficiency in the pin mixing section have been done by Yao et al. [21, 22]. Hwang et al. [23] studied a similar pin region with a quasi-3D analysis technique. A comparable analysis was applied to study the mixing efficiency of chaotic screws $[12,24]$. Dhanasekharan and Kokini [25] used 3D numerical simulations of dough flow in a single screw extruder to demonstrate a scaling-up methodology.

A range of mixing characteristics has been developed in the past to assess mixing quality. Most frequently used to measure the degree of mixing in a system are gross uniformity and intensity of segregation [1], scale of segregation [26], and striation thickness [27]. Prediction of the minor component spatial distribution inside the extruder by using a particle tracking technique in numerical simulations, allows for mixing characterization through pairwise correlation functions [28, 29]. Galaktionov et al. [30, 31] used a mapping method to describe the repeated transport of fluid from one domain to another and to compute the concentra- 
TABLE 1. Extruder design parameters.

\begin{tabular}{cccc}
\hline & Barrel & & \\
$\begin{array}{l}\text { Lead distance } L s \\
(\mathrm{~cm})\end{array}$ & $\begin{array}{c}\text { diameter } \\
D_{b}(\mathrm{~cm})\end{array}$ & $\begin{array}{c}\text { Flight thickness } \\
e(\mathrm{~cm})\end{array}$ & $\begin{array}{c}\text { Flight clearance } \\
\delta_{f},(\mathrm{~cm})\end{array}$ \\
\hline 6.4189 & 6.35 & 0.79375 & 0.0254 \\
\hline
\end{tabular}

tion distribution and other mixing measures. Recently Renyi and Shannon entropies were used to quantify rigorously distributive mixing in polymer processing equipment [32, 33].

One aspect of distributive mixing, namely color homogeneity, is an important factor in determining the quality of the final product in a variety of sectors. As color homogeneity, or in more general terms, property homogeneity, is achieved (through mixing), its assessment becomes a tool in evaluating mixing efficiency in processing and facilitates equipment design optimization. Analysis of color homogeneity in real samples is done by conventional qualitative visual inspection or by spectroscopic methods. In numerical simulations, the coordinates of colored particle tracers can be directly computed and therefore direct quantitative measures of color homogeneity can be applied.

We have proposed [34] the use of Shannon entropy as a tool for color homogeneity analysis of experimental extrusion samples. As we have demonstrated, it can successfully be applied to polymer processing as a tool for in situ analysis of mixing quality. We now want to concentrate on its applicability towards evaluation of color homogeneity in numerical simulations of mixing of color particle species.

\section{NUMERICAL PROCEDURE}

\section{Flow Problem Formulation}

An eight-pitch extruder mesh matching the metering section of a conventional industrial extruder was designed. The mesh consisted of 167704 elements. Table 1 shows some of the important design parameters for the mesh. The overall design of the mesh and a detailed view are shown in Fig. 1a and b. The governing flow equations, equation of continuity (Eq. l) and equation of motion (Eq. 2) have been solved for a power law fluid by using the computational fluid dynamics package FIDAP (www.fluent.com/software/ fidap/). Fully-developed flow, isothermal, and steady-state conditions were assumed.

$$
\underline{\nabla} \cdot \underline{\nu}=0
$$

$$
\rho \frac{D \underline{\underline{\nu}}}{D t}=-\underline{\nabla} P-[\underline{\nabla} \cdot \underline{\underline{\tau}}]
$$

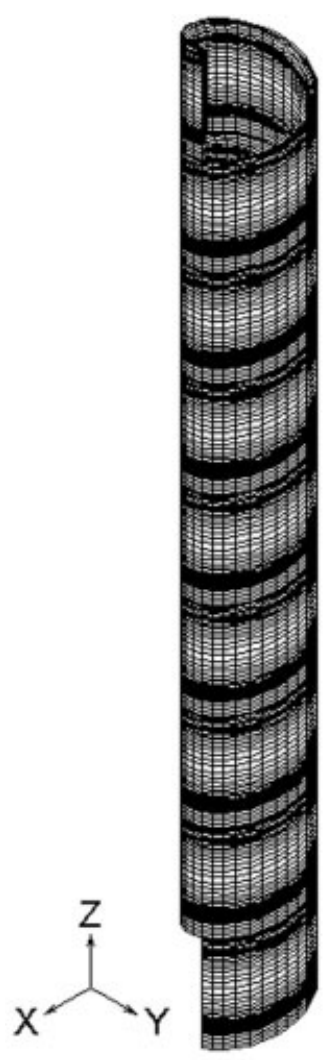

(a)

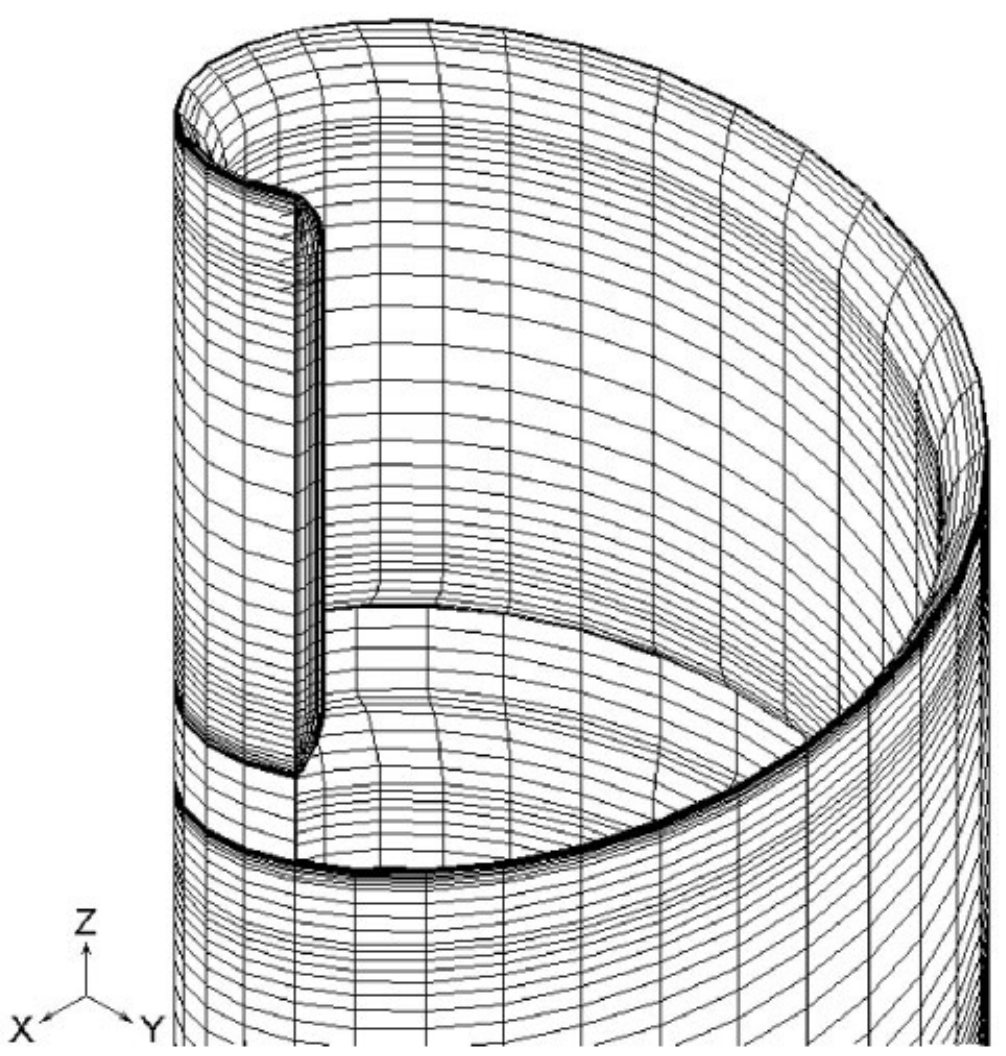

(b)

FIG. 1. Mesh design. a: Overall. b: Detailed. 
TABLE 2. Fluid rheological parameters.

\begin{tabular}{lcc}
$\begin{array}{l}\text { Newtonian viscosity } \mu_{0} \\
(\mathrm{~Pa} \cdot \mathrm{s})\end{array}$ & Power law index $n$ & $\begin{array}{c}\text { Critical shear rate } \\
\dot{\gamma}_{0}\left(\mathrm{~s}^{-1}\right)\end{array}$ \\
\hline 13333 & 0.33 & 15 \\
\hline
\end{tabular}

The parameters characterizing the power law rheological behavior of the fluid matched those for acrylonitrile-butadiene-styrene copolymer (ABS) MAGNUM ${ }^{\mathrm{TM}}$ 3325-MT (trademark of Dow Chemical Co.) resin at $260^{\circ} \mathrm{C}$. The Newtonian viscosity, power law index and the critical shear rate for onset of shear thinning behavior for this resin are shown in Table 2.

\section{Simulation of Color Mixing}

To validate the simulation accuracy, we compared extrusion rates obtained at three different rotational speeds with those observed experimentally. Table 3 shows the values for RPM and pressure difference as boundary conditions for the simulations for each set. The comparison shows good correlation between the experimental data and the simulation results with the error lying within the $8 \%$ range (Table 4). The last column in the table shows the values for the throttle ratio, i.e., the ratio between the pressure flow rate $\left(Q_{p}\right)$ and drag flow rate $\left(Q_{d}\right)$. The value for the drag flow was obtained by running a preliminary simulation with no pressure difference between the inlet and the outlet of the extruder. When a pressure difference between inlet and outlet was applied, the pressure component of the flow was obtained by subtracting the drag component from the overall flow rate. Within the selected range of pressure differences, the pressure flow significantly affects the overall flow rate in the case of 30 RPM (up to almost one-third of the drag flow). Its influence, however, becomes less pronounced in the case of 60 RPM, and almost negligible at 90 RPM.

To analyze the dynamics of particle motion, distribution, and intermixing, a particle tracking procedure was employed. We employed this procedure for the simulation results obtained at 60 RPM to match with the experimental conditions for the screw-crash experiment as described in our previous work [35]. Four pitches in the middle section of the mesh (Fig. 2) have been used for the particle tracking to avoid errors related to the less precise flow field solution at the inlet and the outlet sections of the extruder. Two different particle populations (blue and yellow species) have

TABLE 3. Boundary conditions.

\begin{tabular}{ccc}
\hline Set number & RPM & Pressure difference $\Delta P(\mathrm{MPa})$ \\
\hline 1 & 30 & 10.76 \\
2 & 60 & 4.65 \\
3 & 90 & 2.79 \\
\hline
\end{tabular}

TABLE 4. Experiment vs. simulation: extrusion rate comparison.

\begin{tabular}{ccccc}
\hline & \multicolumn{2}{c}{ Extrusion rate $Q(\mathrm{~g} / \mathrm{s})$} & & \\
\cline { 2 - 3 } Set number & Experiment & Simulation & Error (\%) & $\begin{array}{c}\text { Throttle } \\
\text { ratio } Q_{p} / Q_{d}\end{array}$ \\
\hline 1 & 8.19 & 8.84 & 7.9 & +0.29 \\
2 & 15.88 & 15.12 & 4.8 & +0.1 \\
3 & 22.93 & 21.7 & 5.4 & +0.05 \\
\hline
\end{tabular}

been tracked in order to examine the degree of color intermixing within the extruder.

Figure 3 shows the color pattern of the ABS extrudate cut in the metering section of the extruder obtained during the screw-crash experiment, which was chosen as the initial color particle distribution for the particle tracking procedure. While it is not possible to distinguish between yellow and blue particles in the green region of the cut, we assume that the green color is best represented by a random distribution of yellow and blue particles in combination with each other. We neglected a big dark blue spot on the picture, which was attributed to an unmelted pellet of blue ABS resin. We also neglected the defect in the left portion of this slice, as it was attributed to an air bubble formed in the melt. Thus the initial distribution of colored ABS resin was assumed to be only yellow in the left half of the slice and only green in the right half. To mimic this color distribution we randomly placed 20000 yellow particles across the whole channel of the extruder, and only 1000 blue particles filling out just the right half of the channel (at the pushing flight). A ratio of 20:1 yellow to blue particles was kept to match the same ratio between yellow and blue ABS resin concentrates in the experiment. Figure 4 shows the initial position of blue and yellow particles relative to the mesh of the extruder.

Equation 3 was used to calculate particle position at each consecutive time step.

$$
\underline{X}\left(t_{1}\right)=\underline{X}\left(t_{0}\right)+\int_{t_{0}}^{t_{1}} \underline{V}(t) d t
$$

Here $\underline{X}\left(t_{0}\right)$ is the position of the particle at time $t_{0}, \underline{X}\left(t_{1}\right)$ is the position of the particle at time $t_{1}=t_{0}+d t, \underline{V}(t)$ is particle velocity vector, and $d t$ is the integration time step. The integration time step was chosen to be half of the time needed to cross the smallest element of the mesh in any direction $(X, Y$, or $Z)$ with the highest velocity in that direction $\left(V_{x}, V_{y}\right.$, or $\left.V_{z}\right)$. We employed a 4th order RungeKutta integration procedure [36] to obtain particle positions according to Eq. 3. We assumed particles to be massless points (tracers) with no interaction between themselves or with the carrying fluid. We also assumed continuous (steady-state) injection of the particles at the initial position. 


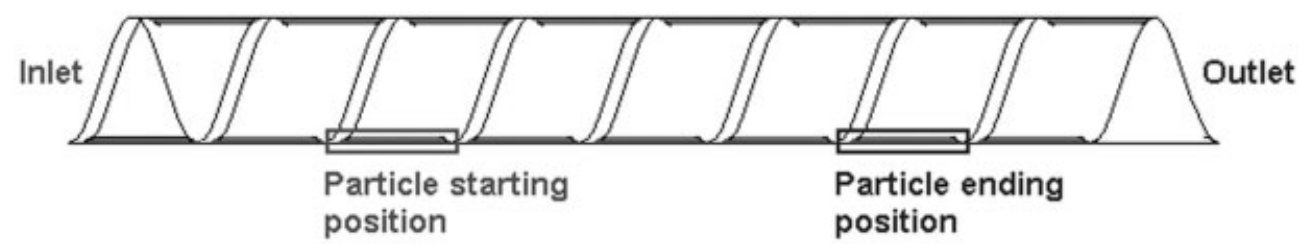

FIG. 2. Middle section of the mesh chosen for particle tracking.

\section{DISCUSSION}

\section{Numerical Results: Particle Distributions}

To monitor particle spatial distributions inside the screw channel we looked at snapshots of intersections of particle trajectories with the $\mathrm{ZX}$ and $\mathrm{ZY}$ planes, starting from the initial particle distribution ( $\mathrm{ZX}$ plane, $Y=0, X>0$ ). Figure 5 shows the snapshots of particle distribution at these planes (slices) along 4 consecutive pitches of the extruder. Circulatory motion of the particles in the screw channel, as described by Ottino et al. [27], generates striations between the yellow and blue tracers, which decrease in thickness along the channel. The mixing becomes more complex in the flight regions due to the complex geometry of the flights.

Figure 6 shows a comparison between the particle tracers distributions obtained by particle tracking with the correspondent polymer cuts from the screw-crash experiment. While the experimentally obtained samples show more complex mixing features compared to the numerical results, one can identify similar trends when looking at the striation formation in both. These similarities are particularly pronounced at the corners, where tracers reorient when approaching the flights. Differences might be due to the presence of air bubbles in the experiment, while the model does not account for them.

\section{Entropy Analysis of Color Mixing}

We analyzed the quality of color intermixing between the yellow and blue particles with a measure defined using informational Shannon entropy. The Shannon entropy is determined by probability distribution as shown in Eq. 4:

$$
S=-\sum_{j=1}^{M} p_{j} \ln p_{j}
$$

Here $M$ is the total number of the outcomes and $p_{j}$ is the probability of outcome $j$ [37]. With respect to mixing, $M$ can be defined as the total number of spatial locations or bins in the system a particle can occupy, and $p_{j}$ is the particle concentration in a bin $j$. The number of bins chosen to describe the quality of distributive mixing defines the scale of observation (the "magnifying glass") at which one can look to the system. The smaller the number of bins is, the larger the scale of observation at which an external observer evaluates the quality of particle distribution. Thus at the largest scale, when only one bin is used to describe our system $(M=1)$, mixing will always appear to be ideal. On the other hand, at a small scale $(M>>1)$ one can see how particle distribution differs from the ideal one on microscopic levels of observation. In view of the fact that we estimate probabilities by using frequencies we need to have a large number of particles in the bins. Hence we consider only the case for which: $\mathrm{N} \geq \mathrm{M}>>1$.

The Shannon entropy can be easily extended to describe a system associated with joint probabilities for multiple events. Thus if those probabilities are defined through particle spatial position $j$ out of $M$ available positions, and color $c$ out of $C$ color species, we can write Shannon formula as shown in Eq. 5:

$$
S=-\sum_{c=1}^{C} \sum_{j=1}^{M} p_{c, j} \ln p_{c, j}
$$

where $p_{c, j}$ is the joint probability for a particle to be of a color " $c$ " and in bin " $j$ ". While the maximum number of bins for an individual particle population is limited by the total number of particles in this population, in the case of multiple particle populations, the maximum number of bins should not exceed the number of particles in the smallest population. In our case this number is equal to 1000 to match the number of blue particle tracers employed in the simulation.

The joint probability for a particle to be located in bin $j$ and to be of a color $c$ is given by Bayes theorem: $p_{c, j}=p_{c / j}$ $p_{j}$, where $p_{c / j}$ is the probability of finding a particle of a color " $c$ " conditional on the bin " $j$ " and $p_{j}$ is the probability for bin " $j . "$ With this we can write:

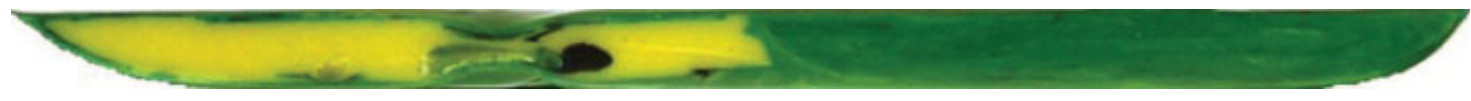

FIG. 3. Experimental sample chosen to describe initial particle distribution. [Color figure can be viewed in the online issue, which is available at www.interscience.wiley.com.] 
FIG. 4. Initial particle position in the mesh. [Color figure can be viewed in the online issue, which is available at www.interscience.wiley. com.]

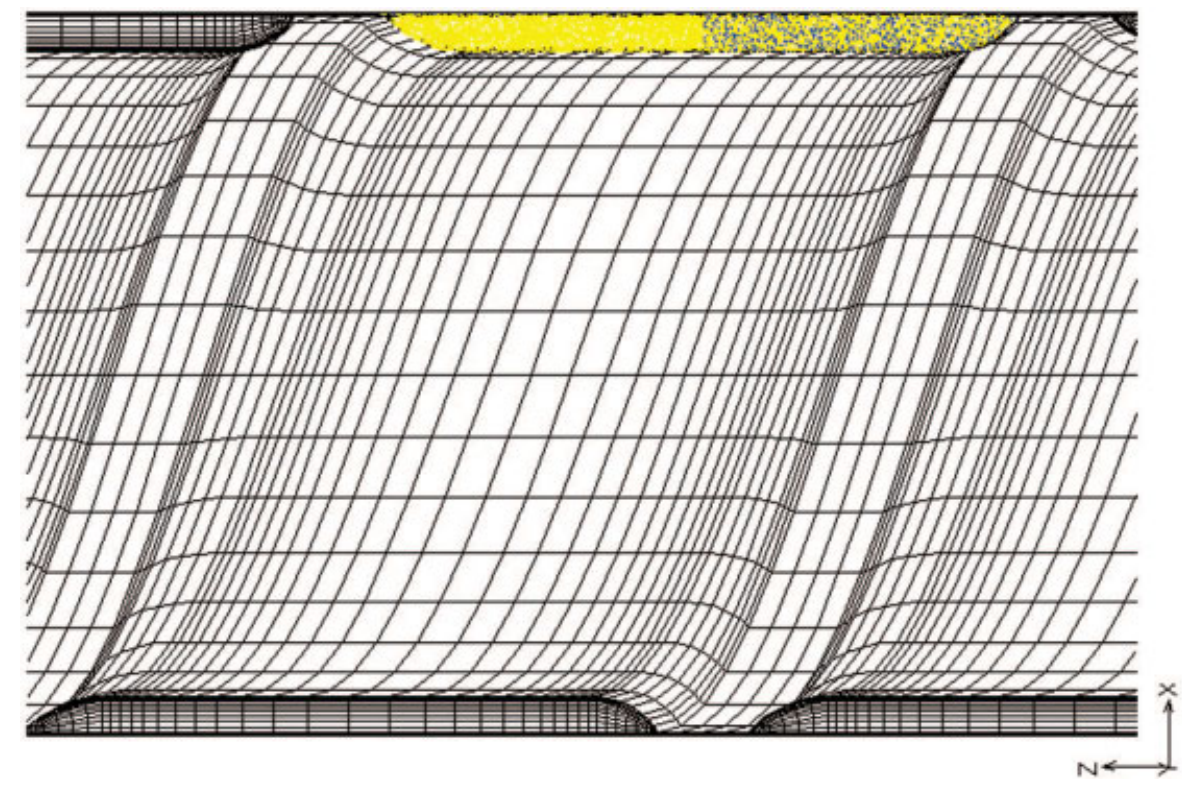

$$
S=-\sum_{c=1}^{C} \sum_{j=1}^{M}\left[\left(p_{c / j} p_{j}\right) \ln \left(p_{c / j} p_{j}\right)\right]
$$

It then follows:

$$
S=-\sum_{j=1}^{M} p_{j} \sum_{c=1}^{C}\left[p_{c / j} \ln p_{c / j}\right]-\sum_{c=1}^{C} p_{c / j} \sum_{j=1}^{M}\left[p_{j} \ln p_{j}\right]
$$

and finally because $\sum_{c=1}^{C} p_{c / j}=1$ for all values of $j$

$$
S=\sum_{j=1}^{M}\left[p_{j} S_{j}(\text { colors })\right]+S(\text { locations })
$$

where

$$
S_{j}(\text { colors })=-\sum_{c=1}^{C}\left[p_{c / j} \ln p_{c / j}\right]
$$
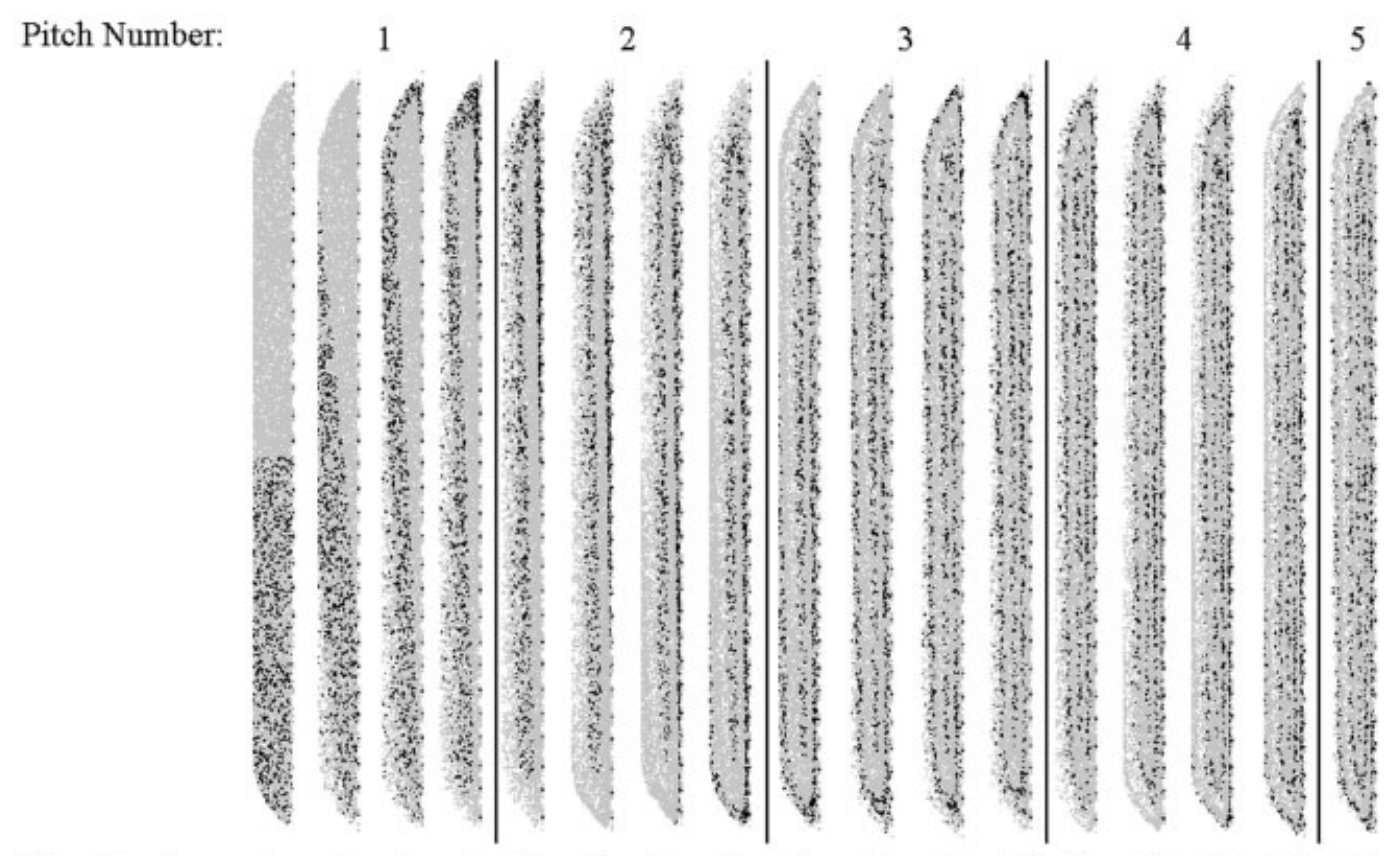

Slice Number:

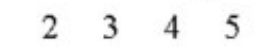

67

10

$\begin{array}{lll}11 & 12 & 13\end{array}$

$\begin{array}{llll}14 & 15 & 16 & 17\end{array}$

FIG. 5. Particle distributions at XZ and YZ cross-sections of the extruder mesh within the four consecutive pitches. 
Slice \#

1
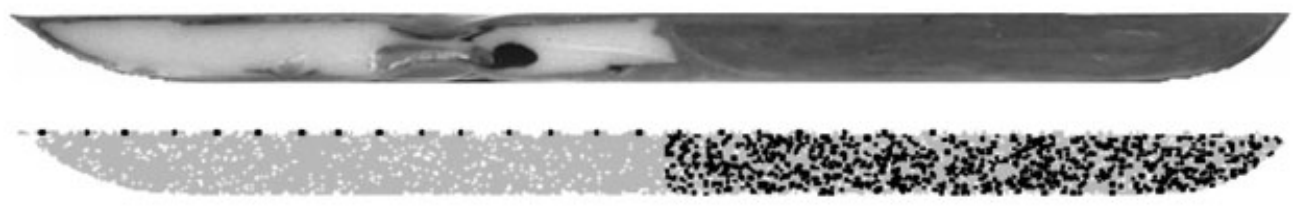

5
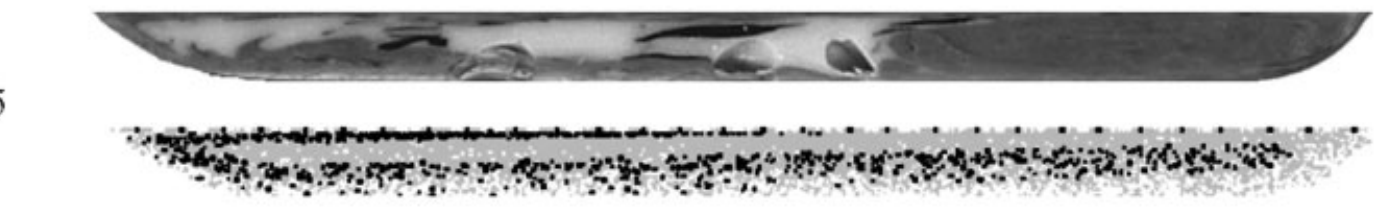

9
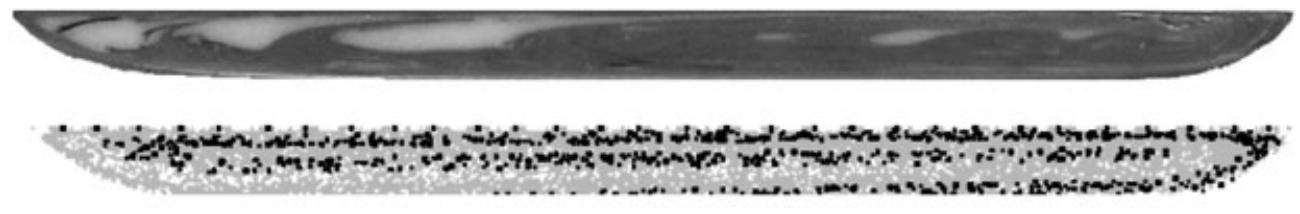

13
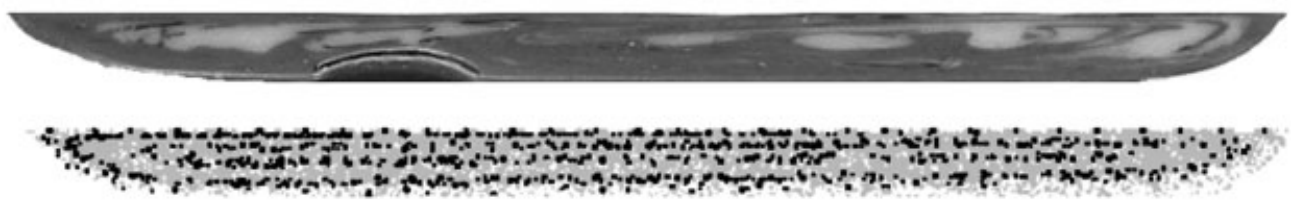

17
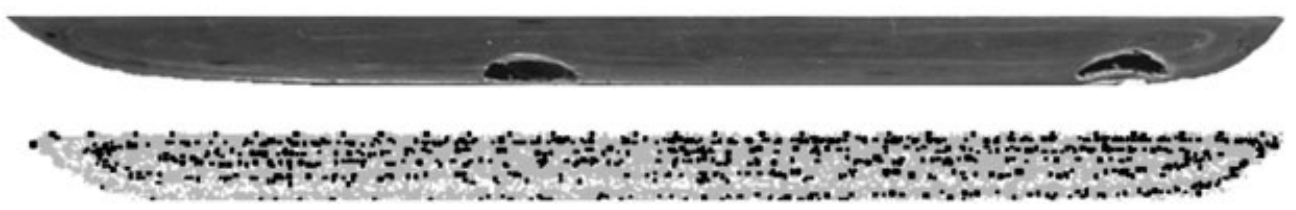

FIG. 6. Comparison between experimental sample cuts and the corresponding numerical slices.

$$
S(\text { locations })=-\sum_{j=1}^{M}\left[p_{j} \ln p_{j}\right] .
$$

$S_{j}$ (colors) is the entropy of color intermixing at the location of bin $j$ and $S$ (locations) is the entropy associated with the overall spatial distribution of particles irrespective of colors.

To account for different relative concentrations of color particle species in the whole system we modify $p_{c / j}$ as follows:

$$
p_{c l j}^{*}=\frac{p_{c l j} / P_{c}}{\sum_{c=1}^{C}\left(p_{c l j} / P_{c}\right)}
$$

Here $P_{c}$ is the relative concentration of particle population $c$ in the whole system. One can think of $P_{c}$ groups of particles of color c and then Eq. 11 provides the probability that a group in bin $\# j$ is of type $c$. This modified probability allows us to find out how different the concentrations of particle species in each bin are compared to their overall relative concentrations $P_{c}$. We compute the entropy of color intermixing at the location of bin $j, S_{j}$ (colors), by using the $p_{c / j}^{*}$ and the Shannon formula (Eq. 9).
Equation 8 can also be written more compactly as:

$$
S=S_{\text {locations }}(\text { colors })+S(\text { locations })
$$

where

$$
S_{\text {locations }}(\text { colors })=\sum_{j=1}^{M}\left[p_{j} S_{j}(\text { colors })\right] .
$$

$S_{\text {locations }}$ (colors) is a spatial average of the entropies associated with color intermixing conditional on location. It is maximized for the particular homogeneous state characterized by: $p_{c / j}^{*}=1 / C$ in each bin " $j$ " ranging from 1 to $M$. The maximum value of $S_{\text {locations }}$ (colors) is equal to $\ln C$. Therefore we normalize this spatial average entropy by $\ln C$ to get values between 0 and 1 . Normalized $S_{\text {locations }}($ colors) show the level of color system homogeneity ranging from 0 (no color intermixing) to 1 (ideal intermixing, uniform hue/ color). Thus we define the normalized $S_{\text {locations }}($ colors) as an index of color homogeneity: 


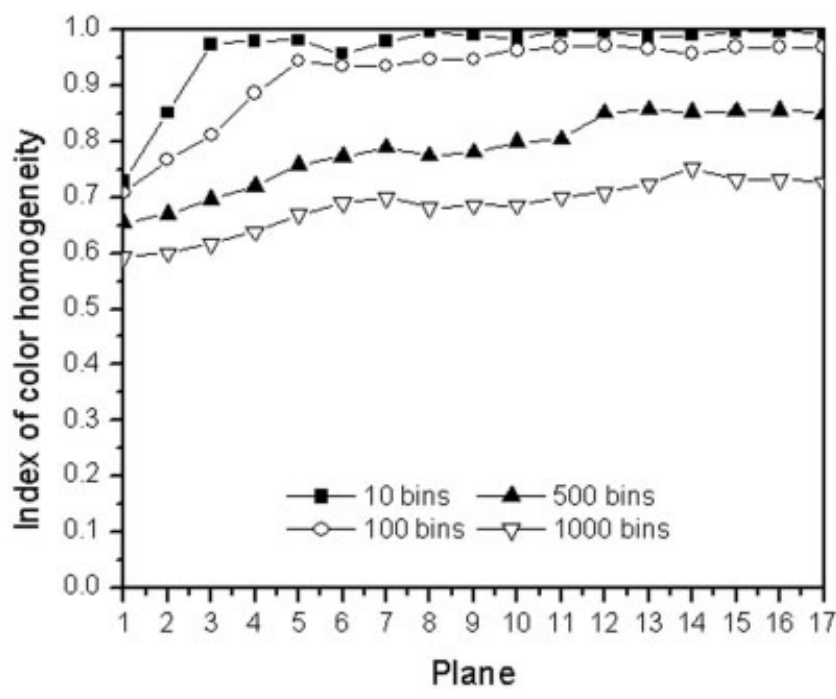

FIG. 7. Evolution of color homogeneity index along the extruder (numerical simulations).
Index of color homogeneity $=\frac{S_{\text {locations }}(\text { colors })}{\ln C}$.

The results of the analysis for the seventeen slices shown in Fig. 5 are displayed in Fig. 7. We used four different numbers of bins $M$ for the analysis, starting with 10 bins and up to 1000 bins, to describe mixing at different scales of observation. The initially high values of the index are explained by a very good intermixing between yellow and blue particles in the right half of the channel of the initial slice. As the mixing progresses, the index shows a trend of increasing color species intermixing at all scales of observation. At the largest scale $(M=10)$ the index shows a very fast increase as blue particles start occupying all ten bins within just 3 slices. Figure 8 shows the distribution of $p_{c / j}^{*}$ for the yellow and blue particle tracers over 10 bins at the 4 first slices. The distribution on the right hand side shows the ideal distribution at $p_{\text {yellow } / j}^{*}=p_{\text {blue/ } j}^{*}=1 / 2$. On the first slice, blue particles are mostly distributed over the half of

FIG. 8. Distribution of modified conditional probabilities $p_{c / j}^{*}$ over 10 bins for slices $1-4$.
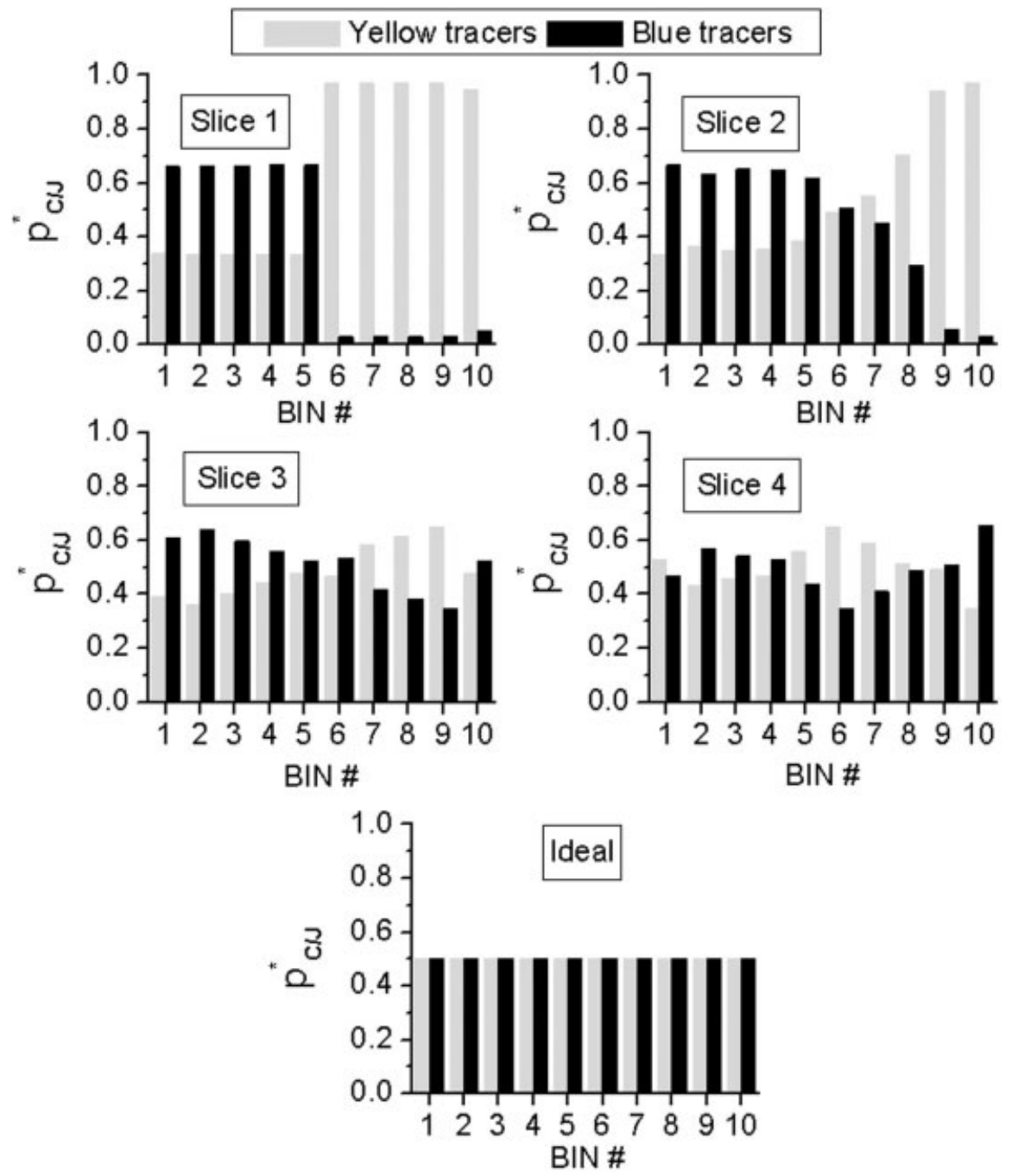


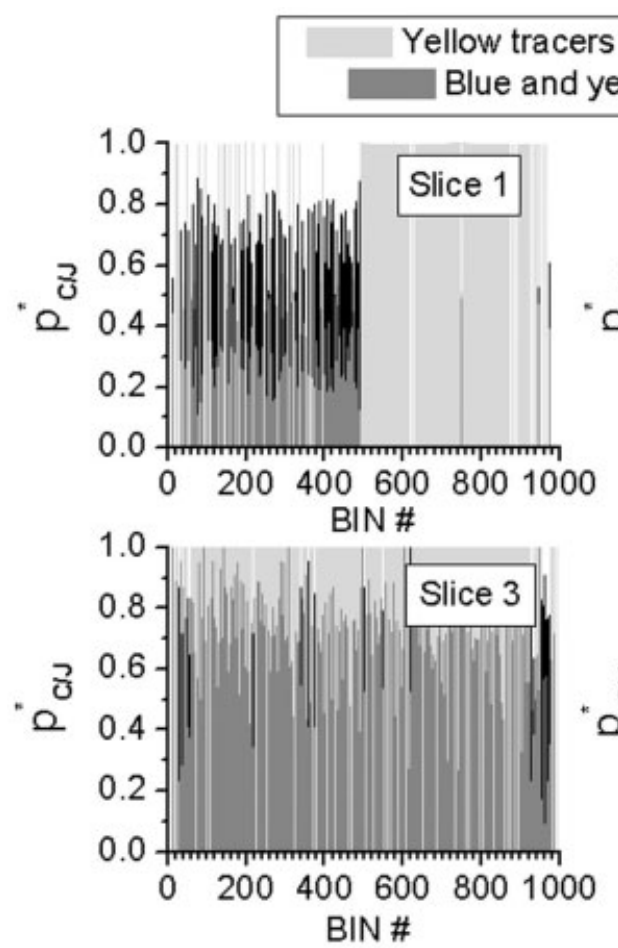

BIN \#

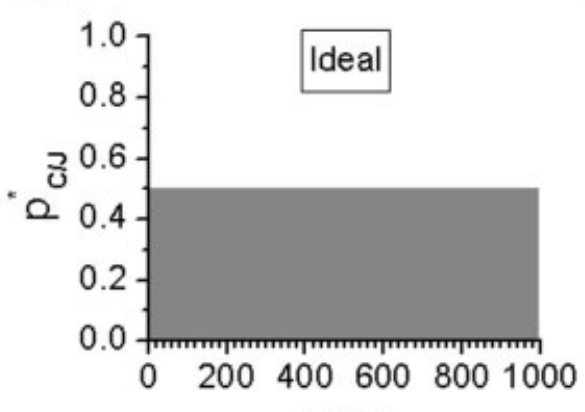

BIN \#
Blue tracers

3
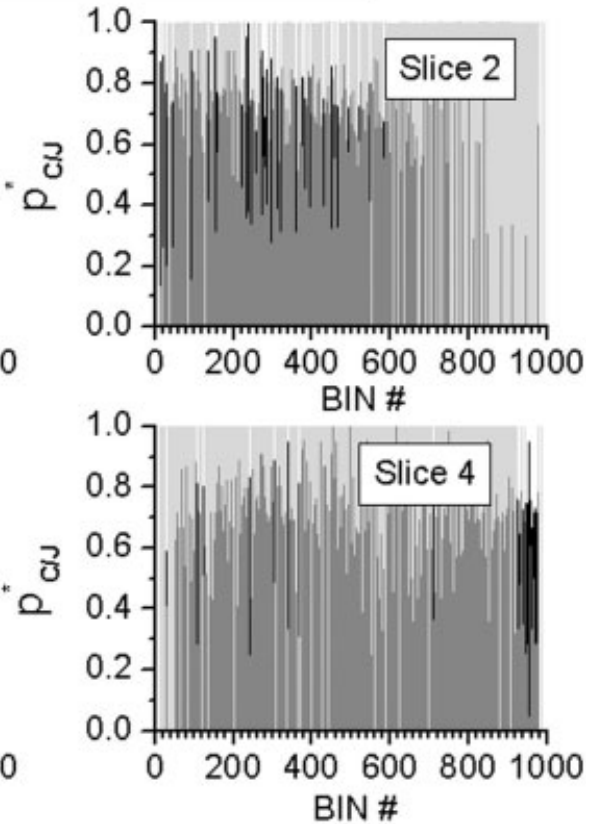

BIN \#

FIG. 9. Distribution of modified conditional probabilities $p_{c / j}^{*}$ over 1000 bins for slices $1-4$. Note: Due to the large number of bins used to represent the plots, some overlapping occurs for the bars referring to the conditional probabilities for blue and yellow tracers.

the channel within 5 bins. Slices 2-4 show how blue particles rapidly redistribute over the other 5 bins, bringing the modified conditional probabilities for yellow and blue species closer to the ideal ones.

At smaller scales $(M=1000)$, however, the index has room to increase further, as there are still many bins occupied solely by the yellow particle tracers as shown in Fig. 9. The difference between these results in fact emphasizes the importance of the scale of the observation. While at a large scale (such as the visual scale) mixing efficiency might appear satisfactory already in the earlier stages of the process, on a more microscopic level, within the first four pitches mixing is shown to be still inappropriate.

\section{Comparison Between the Experimental and Numerical Analyses}

In our previous work [35] we have analyzed extrudate slices obtained from a screw-crash experiment using a similar index of color homogeneity. The experimental samples were analyzed using image analysis and employing either grayscale or red-green-blue (RGB) intensity maps. Each pixel in these maps was characterized by the relative concentrations of black and white particles from a total of 255 particles in each pixel. We chose an ideal color and calculated the index of color homogeneity with reference to the ideal. Figures 10 and 11 show the grayscale images of the experimental samples and the dynamics of the color homogeneity index along the extruder line.

Although the experimental and the numerical entropy analyses (Figs. 11 and 7, respectively) were based on different procedures, the dynamics of mixing as described through the index of color homogeneity is somewhat similar. These results emphasize the potential use of numerical simulations to emulate experimental data and to provide a means for process and design optimization.

\section{CONCLUSIONS}

We conducted numerical simulations of mixing for two colored particle populations within four pitches of the metering section of a conventional single screw extruder. The 


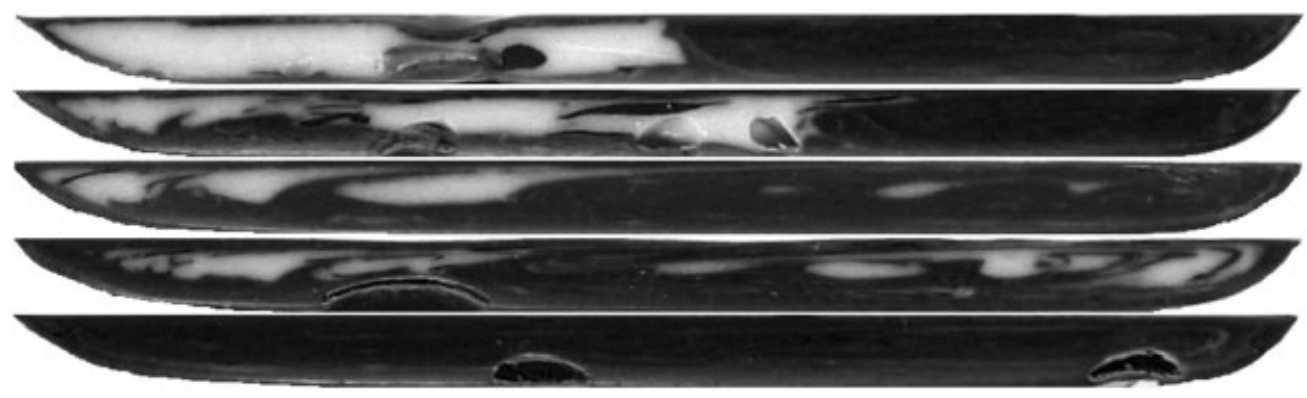

FIG. 10. Grayscale images of the experimental slices.

results of the simulation were interpreted in terms of particle spatial distributions over ZX and ZY slices along the extruder helix. As shown in the past by other authors, mixing in the extruder occurs primarily due to the circulatory motion of particles inside the channel. This motion results in striation formation between the colored populations with a progressive decrease in the striation thickness along the extruder channel.

Mixing patterns obtained from experiments exhibit a more complex behavior. However, a visual qualitative comparison with the particle tracking results shows good agreement in terms of the mode of mixing emphasizing a decrease in the striation thickness. Also we observe similarities between mixing patterns at the corners where particles reorient approaching the flights.

To assess the dynamics of mixing between two colored particle populations we employed an entropic measure, namely the index of color homogeneity. As the index is susceptible to a chosen scale of observation, it can give insight into mixing quality both at macro and microscopic levels. This index can be successfully used in color mixing evaluation and can be potentially applied for scale-up, control, and optimization of mixing equipment.

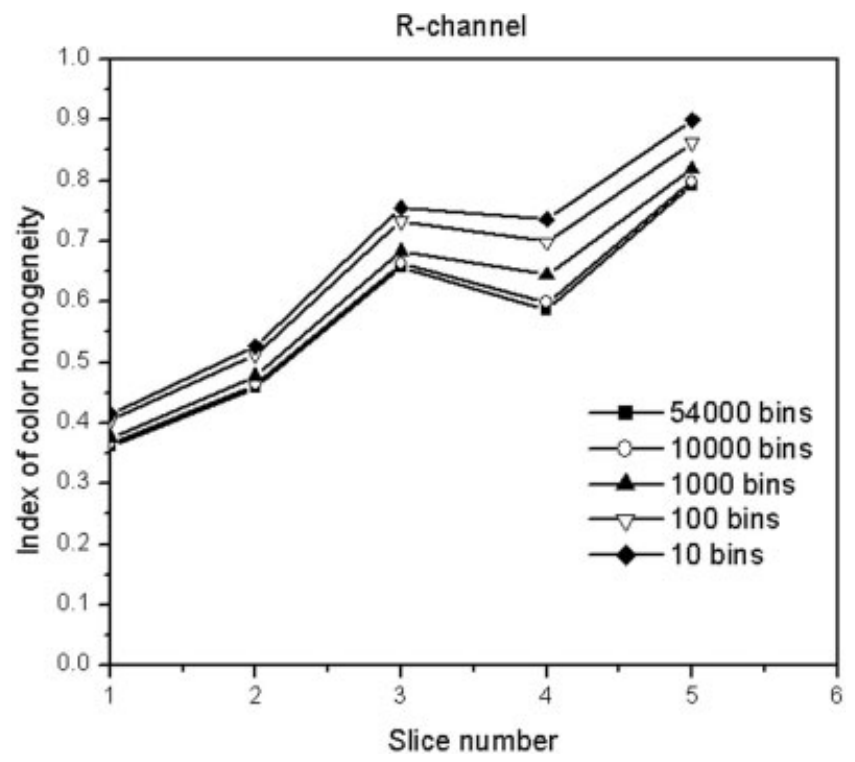

FIG. 11. Evolution of color homogeneity index along the extruder (experimental data).

\section{ACKNOWLEDGMENTS}

We thank Dow Chemical Co. for providing technical assistance in the color mixing experiments, and Ohio $\mathrm{Su}-$ percomputer Center for the use of computing facilities. We have benefited from helpful discussions with Marco Camesasca.

\section{REFERENCES}

1. Z. Tadmor and C.G. Gogos, Principles of Polymer Processing, John Wiley \& Sons, New York (1979).

2. B.H. Maddock, SPE J., 15(5), 383 (1959).

3. Z. Tadmor and I. Klein, Engineering Principles of Plasticating Extrusion, Krieger Publishing Company, Malabar, Florida (1978).

4. G. Lidor and Z. Tadmor, Polym. Eng. Sci., 16, 450 (1976).

5. R.T. Fenner, Polymer, 18, 617 (1977).

6. H. Lappe and H. Potente, SPE-ANTEC Tech. Pap., 174 (1983).

7. C. Rauwendaal, SPE-ANTEC Tech. Pap., 30 (1985).

8. M. Gupta and T.H. Kwon, Polym. Eng. Sci., 30, 1420 (1990).

9. H. Benkreira, R.W. Shales, and M.F. Edwards, Int. Polym. Process., 7, 126 (1992).

10. R.A. Barr and C.A. Chung, U.S. Patent 3,487,503 (1970).

11. R.V. DeBoo and C.B. Heard, U.S. Patent 3,762,693 (1973).

12. T.H. Lee and T.H. Kwon, Adv. Polym. Technol., 18, 53 (1999).

13. G.M. Gale, U.S. Patent 4,419,014 (1983).

14. G.M. Gale, SPE ANTEC Tech. Pap., 109 (1983).

15. R. Brzoskovski, J.L. White, W. Szydlowski, N. Nakajima, and K. Min, Int. Polym. Proc., 3, 134 (1988).

16. Y. Jabushida, R. Brzoskovski, J.L. White, and N. Nakajima, Int. Polym. Proc., 4, 219 (1989).

17. F.E. Dulmage, U.S. Patent 2,753,595 (1956).

18. R.L. Saxton, U.S. Patent 3,006,029 (1961).

19. W. L. Kruger, Plast. Eng., 27 (10), 35 (1981)

20. S.A. Somers, M.A. Spalding, J. Dooley, and K.S. Hyun, SPE-ANTEC Tech. Pap., 222 (1995).

21. W.G. Yao, K. Takahashi, K. Koyama, and G.C. Dai, Chem. Eng. Sci., 52, 13 (1997).

22. W.G. Yao, S. Tanifuji, K. Takahashi, and K. Koyama, Polym. Eng. Sci., 41, 908 (2001). 
23. W.R. Hwang, K.W. Kang, and T.H. Kwon, AIChE J., 50, 1372 (2004).

24. W.R. Hwang and T.H. Kwon, Polym. Eng. Sci., 40, 702 (2000).

25. K.M. Dhanasekharan and J.L. Kokini, J. Food Eng., 60, 421 (2003).

26. P.V. Danckwerts, Appl. Sci. Res. A, 3, 279 (1953).

27. J.M. Ottino, The Kinematics of Mixing: Stretching, Chaos, and Transport, Cambridge University Press, Cambridge, UK (1989).

28. H.H. Yang, T. Wong, and I. Manas-Zloczower, "Flow Field Analysis of a Banbury Mixer," in Mixing and Compounding of Polymers, I. Manas-Zloczower and Z. Tadmor, eds., Hanser Publishers, Munich-Vienna-New York, 189 (1994).

29. T. Avalosse, Macromol. Symp., 112, 91 (1996).

30. O.S. Galaktionov, P.D. Anderson, P.G.M. Kruijt, G.W.M. Peters, and H.E.H. Meijer, Comput. Fluids, 30(3), 271 (2001).
31. O.S. Galaktionov, P.D. Anderson, G.W.M. Peters, and H.E.H. Meijer, Int. J. Num. Methods Fluids, 40(3-4), 345 (2002).

32. W. Wang, I. Manas-Zloczower, and M. Kaufman, Int. Polym. Process., 16, 315 (2001).

33. W. Wang, I. Manas-Zloczower, and M. Kaufman, AICHE J., 49, 1637 (2003).

34. K. Alemaskin, M. Camesasca, I. Manas-Zloczower, M. Kaufman, E.K. Kim, M.A. Spalding, W.A. Trumbull, and R.D. Swain, SPE-ANTEC Tech. Pap., 167 (2004).

35. K. Alemaskin, M. Camesasca, I. Manas-Zloczower, and M. Kaufman, Polym. Eng. Sci., in press.

36. W.H. Press, S.A. Teukolsky, W.T. Vetterling, and B.P. Flannery, Numerical Recipes in FORTRAN: The Art of Scientific Computing, Cambridge University Press, Cambridge, UK (1992).

37. C.E. Shannon, Bell Syst. Techn. J., 27, 379 (1948).

Post-print standardized by MSL Academic Endeavors, the imprint of the Michael Schwartz Library at Cleveland State University, 2017. 